\title{
REGULARITY AND UNITARITY OF AFFINE AND HYPERBOLIC TIME-FREQUENCY REPRESENTATIONS*
}

\author{
Franz Hlawatsch ${ }^{1}$ Antonia Papandreou ${ }^{2}$ G. Faye Boudreaux-Bartels ${ }^{2}$ \\ ${ }^{1}$ Inst. für Nachrichtentechnik und Hochfrequenztechnik, Technische Universität Wien, A-1040 Vienna, Austria \\ ${ }^{2}$ Dept. of Electrical Engineering, University of Rhode Island, Kingston, RI 02881 USA \\ email addresses: fhlawatsQemail.tuwien.ac.at, antoniaQele.uri.edu, boudQele.uri.edu
}

\begin{abstract}
The affine and hyperbolic classes of quadratic timefrequency representations (QTFRs) provide frameworks for multiresolution or constant-Q time-frequency analysis. This paper studies the QTFR properties of regularity (QTFR reversibility) and unitarity (preservation of inner products, Moyal's formula) in the context of affine and hyperbolic QTFRs. We develop the calculus of inverse kernels and discuse important implications of regularity and unitarity, such as signal recovery, the derivation of other quadratic signal representations, optimum detection, leastsquares signal synthesis, the effect of linear signal transforms, and the construction of QTFR basis systems.
\end{abstract}

\section{INTRODUCTION}

The recently introduced affine class $[1,2,3]$ and hyperbolic class $[4,5,6]$ of quadratic time-frequency representations (QTFRs) provide appropriate frameworks for a multiresolution or constant- $Q$ type of energetic time-frequency (TF) analysis. Hence, these QTFR classes are useful for Doppler-invariant signals used in bat and dolphin echolocation [7], and self-similar signals used in fractals and fractional Brownian motion [8]. Moreover, constant-Q TF analysis is roughly consistent with human auditory perception above $600 \mathrm{~Hz}$.

For any QTFR, the properties of regularity (QTFR reversibility) and unitarity (preservation of inner products, Moyal's formula) are of fundamental importance [9]. These properties have far-reaching implications concerning the recovery of the signal, the derivation of other quadratic signal representations, the QTFR's reaction to linear signal transforms, a TF formulation of optimum detection, the applicability of standard methods for least-squares signal synthesis, and the construction of QTFR basis systems. In [9], the theory of regularity and unitarity has been developed and specialized to Cohen's class. In this paper, we shall consider the regularity and unitarity properties in the context of the affine and hyperbolic classes.

The Affine Class. The affine class (AC) comprises all QTFRs $T_{x}(t, f)$ that are invariant (or "covariant") to time shifts and TF scalings $[1,2,3]$,

$$
\tilde{X}(f)=e^{-j 2 \pi r f} \sqrt{a} X(a f) \Rightarrow T_{\tilde{z}}(t, f)=T_{x}\left(\frac{t-\tau}{a}, a f\right) .
$$

Any QTFR $T_{z}(t, f)$ that is a member of the AC can be written as

$$
T_{x}(t, f)=\frac{1}{|f|} \int_{f^{\prime}} \int_{\nu} \Gamma_{x}\left(\frac{f^{\prime}}{f}, \frac{\nu}{f}\right) U_{x}\left(f^{\prime}, \nu\right) e^{j 2 \pi t \nu} d f^{\prime} d \nu
$$

- Funding by ONR grants N00014-89-J-1812, N00014-92-J1499 and by Grants P7354-PHY and J0530-TEC of the Fond zur Förderung der wissenschaftlichen Forschung. with the signal product $U_{x}(f, \nu)=X\left(f+\frac{\nu}{2}\right) X^{*}\left(f-\frac{\nu}{2}\right)$. Here, $t$ and $f$ denote time and frequency, respectively, $x(t)$ is the signal under analysis, $X(f)$ is the signal's Fourier transform, and integrals typically go from $-\infty$ to $\infty$ unless specified otherwise. The above "normal form" shows that $T_{x}(t, f)$ can be derived from the signal product $U_{x}(f, \nu)$ by a characteristic linear transform involving a two-dimensional kernel function $\Gamma_{T}(b, \beta)$. Some members of the $A C$ are the Wigner distribution, the ChoiWilliams distribution, the scalogram (squared magnitude of the wavelet transform), and the Bertrand distribution.

The Hyperbolic Class. The hyperbolic class (HC) comprises all QTFRs $T_{x}(t, f)$ that are invariant to "hyperbolic time shifts ${ }^{x}$ and $T F$ scalings $[4,5,6]$,

$$
\tilde{X}(f)=e^{-j 2 \pi c \ln f} \sqrt{a} X(a f) \Rightarrow T_{\tilde{z}}(t, f)=T_{x}\left(\frac{t-\frac{c}{f}}{a}, a f\right) .
$$

Assuming a fixed reference frequency $f_{r}>0$, any HC member $T_{x}(t, f)$ can be written as ${ }^{1}$

$$
T_{x}(t, f)=\int_{b} \int_{\beta} \Phi_{r}\left(\ln \frac{f}{f_{r}}-b, \beta\right) V_{x}(b, \beta) e^{j 2 \pi t f \beta} d b d \beta
$$

with the hyperbolic signal product

$$
V_{z}(b, \beta)=f_{r} e^{b} X\left(f_{r} e^{b+\beta / 2}\right) X^{*}\left(f_{r} e^{b-\beta / 2}\right) .
$$

This "normal form" shows that any hyperbolic QTFR can be derived from the hyperbolic signal product $V_{z}(b, \beta)$ by a characteristic linear transform involving a two-dimensional kernel function $\Phi_{T}(b, \beta)$. Some HC members are the AltesMarinovic distribution, the hyperbologram (squared magnitude of the "hyperbolic wavelet trangform"), and the Bertrand distribution.

In both the $A C$ and the $H C$, the TF scaling operator plays a central role. However, while the $A C$ is based on conventional time shifts, the HC uses hyperbolic time shifts which are better matched to TF scalings. The HC can be derived from Cohen's class via a “constant- $Q$ warping" procedure which results in a one-to-one correspondence between the HC and Cohen's class $[4,6]$.

\section{REGULARITY}

A QTFR is regular if the linear transform underlying the normal form can be inverted. In [9], the general theory of regular QTFRs has been developed and applied to Cohen's class. Here, we shall discuss the regularity property for the affine and hyperbolic QTFR classes.

IIn the context of the HC, the signal $x(t)$ is assumed to be analytic, i.e., its Fourier transform $X(f)$ is zero for $f<0$. Accordingly, a hyperbolic QTFR $T_{x}(t, f)$ is defined for $f>0$ only.

III-245

0-7803-0946-4/93 \$3.00@1993 IEEE 
Regularity in the Affine Clase. An affine OTFR $T_{x}(t, f)$ is regular if the normal form (1) can be inverted, i.e., if the signal product $U_{s}(f, \nu)$ can be recovered from $T_{x}(t, f)$ via a linear transform. It can be shown that this inverse linear transform, if it exists at all, has the form

$$
U_{x}(f, \nu)=\frac{1}{|f|} \iint_{f^{\prime}} \Gamma_{T}^{-1}\left(\frac{f^{\prime}}{f}, \frac{\nu}{f}\right) T_{x}\left(t, f^{\prime}\right) e^{-j 2 \pi \nu t} d t d f^{\prime}
$$

with the inverse kernel $\Gamma_{T}^{-1}(b, \beta)$. The inverse kernel is related to the "direct" kernel $\Gamma_{T}(b, \beta)$ according to

$$
\int_{b^{\prime}} \Gamma_{T}^{-1}\left(\frac{b}{b^{\prime}}, \beta b\right) \Gamma_{T}\left(b^{\prime}, \beta b^{\prime}\right) \frac{d b^{\prime}}{\left|b^{\prime}\right|}=\delta(b-1), \quad \forall \beta .
$$

Regularity in the Hyperbolic Class. Similarly, a hyperbolic QTFR $T_{x}(t, f)$ is regular if the normal form (2) can be inverted as

$V_{x}(b, \beta)=\int_{t} \int_{0}^{\infty} \Phi_{T}^{-1}\left(b-\ln \frac{f}{f_{r}}, \beta\right) T_{x}(t, f) e^{-j 2 \pi \beta t f} d t d f$

where the inverse kernel $\Phi_{T}^{-1}(b, \beta)$ is related to the "direct" kernel $\Phi_{\boldsymbol{T}}(b, \beta)$ according to

$$
\int_{b^{\prime}} \Phi_{T}^{-1}\left(b-b^{\prime}, \beta\right) \Phi_{T}\left(b^{\prime}, \beta\right) d b^{\prime}=\delta(b), \quad \forall \beta .
$$

Calculation of the inverse kernels $\Gamma_{T}^{-1}(b, \beta)$ or $\Phi_{T}^{-1}(b, \beta)$ is particularly simple if the QTFR is unitary (see Section 3 ). The regularity of a QTFR has important implications [9], some of which are now summarized.

Signal Recovery. From a regular QTFR $T_{3}(t, f)$, the signal $x(t)$ can be recovered up to a constant phase factor. In the case of an affine QTFR, we first derive the signal product $U_{x}(f, \nu)$ using the inverse normal form (3). Then, the signal's Fourier transform $X(f)$ is obtained as

$$
X(f)=e^{j \phi} U_{x}\left(\frac{f+f^{\prime}}{2}, f-f^{\prime}\right) / \sqrt{U_{x}\left(f^{\prime}, 0\right)},
$$

where $\phi$ is an unknown constant phase and $f^{\prime}$ is any frequency for which $U_{x}\left(f^{\prime}, 0\right) \neq 0$. For a hyperbolic QTFR, we derive $V_{x}(b, \beta)$ via $(5)$ and then obtain $X(f)$ as

$$
X(f)=e^{j \phi} V_{x}\left(\ln \frac{\sqrt{f f^{\prime}}}{f_{r}}, \ln \frac{f}{f^{\prime}}\right) / \sqrt{f V_{x}\left(\ln \frac{f^{\prime}}{f_{r}}, 0\right)},
$$

where $f^{\prime}$ is such that $V_{x}\left(\ln \frac{f^{\prime}}{f_{r}}, 0\right) \neq 0$.

Derivation of Quadratic Signal Representations. From a regular QTFR, any quadratic signal representation can be derived via a linear transform. Any arbitrary quadratic signal representation can be written as [9]

$$
\tilde{T}_{z}(\Theta)=\int_{f_{1}} \int_{f_{2}} K_{\tilde{T}}\left(\Theta ; f_{1}, f_{2}\right) X\left(f_{1}\right) X^{*}\left(f_{2}\right) d f_{1} d f_{2},
$$

where $\theta$ is a parameter or a vector of parameters such as $(t, f)$ in the case of a QTFR, and $K_{*}\left(\theta ; f_{1}, f_{2}\right)$ is some kernel characterizing $\tilde{T}$. Using the inverse normal form (3) or (5), it can be shown that $\tilde{T}_{z}(\Theta)$ can be derived from a regular QTFR $T_{2}(t, f)$ as

$$
\tilde{T}_{x}(\Theta)=\int_{t^{\prime}} \int_{f^{\prime}} L_{\tilde{T} T}\left(\Theta ; t^{\prime}, f^{\prime}\right) T_{x}\left(t^{\prime}, f^{\prime}\right) d t^{\prime} d f^{\prime} .
$$

The construction of the kernel $L_{\tilde{\hat{T} T}}\left(\Theta ; t^{\prime}, f^{\prime}\right)$ depends on the QTFR class. If the original $T_{2}(t, f)$ is a regular affine QTFR, then

$$
\begin{aligned}
& L_{\tilde{T} T}\left(\Theta ; t^{\prime}, f^{\prime}\right)= \\
& \int_{f} \int_{\nu} K_{\tilde{T}}\left(\Theta ; f+\frac{\nu}{2}, f-\frac{\nu}{2}\right) \Gamma_{T}^{-1}\left(\frac{f^{\prime}}{f}, \frac{\nu}{f}\right) e^{-j 2 \pi t^{\prime} \nu} \frac{d f}{|f|} d \nu .
\end{aligned}
$$

If the new $\tilde{T}_{x}(\theta)$ is also affine, then (8) reduces to

$$
\tilde{T}_{x}(t, f)=\int_{t^{\prime}} \int_{f^{\prime}} \lambda_{\tilde{T} T}\left(f\left(t-t^{\prime}\right), \frac{f^{\prime}}{f}\right) T_{x}\left(t^{\prime}, f^{\prime}\right) d t^{\prime} d f^{\prime}
$$

with

$$
\lambda_{\tilde{T} T}(a, b)=\int_{b^{\prime}} \int_{\beta} \Gamma_{\tilde{T}}\left(\frac{b}{b^{\prime}}, \beta\right) \Gamma_{T}^{-1}\left(b^{\prime}, \frac{\beta}{b} b^{\prime}\right) e^{j 2 \pi a \beta} \frac{d b^{\prime}}{\left|b^{\prime}\right|} d \beta .
$$

On the other hand, if the original $T_{x}(t, f)$ is a regular hy perbolic QTFR, then

$L_{T T}\left(\theta ; t^{\prime}, f^{\prime}\right)=$

$\int_{0}^{\infty} \int_{\beta} K_{\tilde{T}}\left(\theta ; f e^{\frac{\beta}{2}}, f e^{-\frac{\beta}{2}}\right) \Phi_{T}^{-1}\left(\ln \frac{f}{f^{\prime}}, \beta\right) e^{-j 2 \pi t^{\prime} f^{\prime} \beta} d f d \beta$.

If the new $\tilde{T}_{x}(\Theta)$ is also hyperbolic, then (8) reduces to

$$
\tilde{T}_{x}(t, f)=\int_{t^{\prime}} \int_{0}^{\infty} \lambda_{\tilde{T} T}\left(t f-t^{\prime} f^{\prime}, \ln \frac{f}{f^{\prime}}\right) T_{x}\left(t^{\prime}, f^{\prime}\right) d t^{\prime} d f^{\prime}
$$

with

$$
\lambda_{\tilde{T} T}(a, b)=\int_{b^{\prime}} \int_{\beta} \Phi_{\tilde{T}}\left(b-b^{\prime}, \beta\right) \Phi_{T}^{-1}\left(b^{\prime}, \beta\right) e^{j 2 \pi a \beta} d b^{\prime} d \beta .
$$

Quadratic Forms and Signal Detection. We next consider the problem of detecting a zero-mean, finiteenergy, Gaussian, nonstationary "signal" process in the presence of some interfering zero-mean, finite-energy, Gaussian, nonstationary "noise" process. It is well known [10] that an optimum detection statistic is given by the quadratic form of the observed signal $X(f)$,

$$
\Lambda_{x}=\int_{f_{1}} \int_{f_{2}} X\left(f_{1}\right) K_{\Lambda}\left(f_{1}, f_{2}\right) X^{*}\left(f_{2}\right) d f_{1} d f_{2},
$$

where the kernel $K_{\Lambda}\left(f_{1}, f_{2}\right)$ depends upon the autocorrelation functions of the signal and noise processes. Since the quadratic form $\Lambda_{x}$ is a special case of a quadratic signal representation in (7), we apply (8) and obtain the following TF formulation of the detection statistic,

$$
\Lambda_{x}=\int_{t^{\prime}} \int_{f^{\prime}} L_{\Lambda T}\left(t^{\prime}, f^{\prime}\right) T_{z}\left(t^{\prime}, f^{\prime}\right) d t^{\prime} d f^{\prime},
$$

where

$L_{\Delta T}\left(t^{\prime}, f^{\prime}\right)=\int_{f} \int_{\nu} K_{\mathrm{A}}\left(f+\frac{\nu}{2}, f-\frac{\nu}{2}\right) \Gamma_{T}^{-1}\left(\frac{f^{\prime}}{f}, \frac{\nu}{f}\right) e^{-j 2 \pi t^{\prime} \nu} \frac{d f}{|f|} d \nu$

for an affine QTFR and

$$
\begin{aligned}
& L_{\Lambda T}\left(t^{\prime}, f^{\prime}\right)= \\
& \int_{0}^{\infty} \int_{\beta} K_{\Lambda}\left(f e^{\frac{\beta}{2}}, f e^{-\frac{\beta}{2}}\right) \Phi_{T}^{-1}\left(\ln \frac{f}{f^{\prime}}, \beta\right) e^{-j 2 \pi t^{\prime} f^{\prime} \beta} d f d \beta
\end{aligned}
$$

for a hyperbolic QTFR. Expression (9) can be interpreted as a TF correlator using a TF reference function $L_{A} r(t, f)$ which incorporates the statistical information about the signal and noise processes [11]. 
Effects of Linear Signal Transforms. For a regular $Q T F R$, a linear tranuform of the signal results in a linear transform of the QTFR. The QTFR $T_{\xi}(t, f)$ of a linearly transformed signal

$$
\tilde{X}(f)=\int_{f^{\prime}} H\left(f, f^{\prime}\right) X\left(f^{\prime}\right) d f^{\prime}
$$

is a linearly transformed QTFR of the original signal,

$$
T_{\tilde{z}}(t, f)=\int_{t^{\prime}} \int_{f^{\prime}} L_{H}\left(t, f ; t^{\prime}, f^{\prime}\right) T_{z}\left(t^{\prime}, f^{\prime}\right) d t^{\prime} d f^{\prime} .
$$

The kernel $L_{H}\left(t, f ; t^{\prime}, f^{\prime}\right)$ is constructed as [9]

$L_{H}\left(t, f ; t^{\prime}, f^{\prime}\right)=$

$\frac{1}{|f|} \int_{f_{1}} \int_{\nu_{1}} \int_{f_{3}} \int_{\nu_{2}} H\left(f_{1}+\frac{\nu_{1}}{2}, f_{2}+\frac{\nu_{2}}{2}\right) H^{*}\left(f_{1}-\frac{\nu_{1}}{2}, f_{2}-\frac{\nu_{2}}{2}\right)$

$\cdot \Gamma_{T}\left(\frac{f_{1}}{f}, \frac{\nu_{1}}{f}\right) \Gamma_{T}^{-1}\left(\frac{f^{\prime}}{f_{2}}, \frac{\nu_{2}}{f_{2}}\right) e^{j 2 \pi\left(t \nu_{1}-t^{\prime} \nu_{2}\right)} d f_{1} d \nu_{1} \frac{d f_{2}}{\left|f_{2}\right|} d \nu_{2}$

in the case of an affine QTFR, and

$L_{H}\left(t, f ; t^{\prime}, f^{\prime}\right)=$

$\int_{0}^{\infty} \int_{\beta_{1}} \int_{0}^{\infty} \int_{\beta_{2}} H\left(f_{1} e^{\frac{\beta_{1}}{2}}, f_{2} e^{\frac{\beta_{2}}{2}}\right) H^{*}\left(f_{1} e^{-\frac{\beta_{1}}{2}}, f_{2} e^{-\frac{\beta_{2}}{2}}\right)$

. $\Phi_{T}\left(\ln \frac{f}{f_{1}}, \beta_{1}\right) \Phi_{T}^{-1}\left(\ln \frac{f_{2}}{f^{\prime}}, \beta_{2}\right) e^{j 2 \pi\left(t f \beta_{1}-t^{\prime} f^{\prime} \beta_{2}\right)} d f_{1} d \beta_{1} d f_{2} d \beta_{2}$

in the case of a hyperbolic QTFR.

Induced QTFR Basis [9]. If $T_{x}(t, f)$ is a regular QTFR, and the signals $x_{k}(t)$ with $k=1, \ldots, K$ are linearly independent, the signals' cross-QTFRs ${ }^{2} T_{x f}(t, f)$ are linearly independent as well. More can be said if $T$ is a regular hyperbolic QTFR. Then, if the $x_{k}(t)$ (with $\left.k=1, \ldots, \infty\right)$ are complete in the linear space of all square-integrable (finiteenergy), analytic signals, the signals' cross-QTFRs will be complete in $L_{2}\left(\mathbb{R} \times \mathbb{R}_{+}\right)$, the linear space of all squareintegrable 2-D functions $F(t, f)$ defined for $f>0$. Hence, any such function can be expanded as

$$
F(t, f)=\sum_{k=1}^{\infty} \sum_{l=1}^{\infty} c_{k l} T_{x_{k}, x_{l}}(t, f),
$$

where the expansion coefficients $c_{k l}$ are uniquely determined for any $F(t, f)$.

If $T$ is a regular affine QTFR, then completeness of the cross-QTFRs requires an additional condition, namely,

$$
\int_{b^{\prime}} \Gamma_{T}^{-1}\left(\frac{b}{b^{\prime}}, \frac{\beta}{b^{\prime}}\right) \Gamma_{T}\left(b^{\prime}, \beta\right) \frac{d b^{\prime}}{\left|b^{\prime}\right|}=\delta(b-1), \quad \forall \beta
$$

(note the difference from (4)).

\section{UNITARITY}

A QTFR is unitary [9] if the linear transform underlying the normal form (1) or (2) preserves inner products. This is equivalent to the validity of Moyal's formula $a^{3}$

${ }^{2}$ The crose-QTFRs are defined by (1) or (2) with $U_{x}(f, \nu)$ and $V_{x}(b, \beta)$ replaced by $U_{x, y}(f, \nu)=X\left(f+\frac{\nu}{2}\right) Y^{*}\left(f-\frac{\nu}{2}\right)$ and $V_{x, y}(b, \beta)=f_{r} e^{b} X\left(f_{r} e^{b+\beta / 2}\right) Y^{*}\left(f_{r} e^{b-\beta / 2}\right)$, respectively.

$$
\left\langle T_{x}, T_{y}\right\rangle=|\langle x, y\rangle|^{2} \text {. }
$$

Unitarity in the Affine Clase. It can be shown that an affine QTFR is unitary if and only if its kernel satisfies

$$
\int_{b^{\prime}} \Gamma_{T}^{*}\left(\frac{b^{\prime}}{b}, \beta b^{\prime}\right) \Gamma_{T}\left(b^{\prime}, \beta b^{\prime}\right) d b^{\prime}=\delta(b-1), \quad \forall \beta .
$$

Comparing with (4), it is seen that a unitary affine QTFR is also regular, with inverse kernel given by

$$
\Gamma_{T}^{-1}(b, \beta)=\frac{1}{|b|} \Gamma_{T}^{*}\left(\frac{1}{b}, \frac{\beta}{b}\right) .
$$

Examples of unitary affine QTFRs are the (generalized) Wigner distribution [12] and the Bertrand distribution [1] (cf. Section 4). Affine QTFRs derived from the Wigner distribution by a smoothing operation (e.g., the scalogram [2]) are non-unitary and non-regular.

Unitarity in the Hyperbolic Class. Similarly, a hyperbolic QTFR is unitary if and only if

$$
\int_{b^{\prime}} \Phi_{T}^{*}\left(b^{\prime}-b, \beta\right) \Phi_{T}\left(b^{\prime}, \beta\right) d b^{\prime}=\delta(b), \quad \forall \beta .
$$

Comparing with (6), we see that a unitary hyperbolic QTFR is regular with inverse kernel

$$
\Phi_{T}^{-1}(b, \beta)=\Phi_{T}^{*}(-b, \beta) .
$$

Examples of unitary hyperbolic QTFRs are the (generalized) Altes-Marinovic distribution and the Bertrand distribution [4]. Hyperbolic QTFRs involving a smoothing operation (e.g., the hyperbologram [4]) are non-unitary and non-regular.

Induced QTFR Basis. If $T_{x}(t, f)$ is a unitary QTFR, and the signals $x_{k}(t)$ with $k=1, \ldots, K$ are orthonormal, then the signals' cross-QTFRs $T_{x_{n}, x_{l}}(t, f)$ are orthonormal as well. In the case of a unitary hyperbolic QTFR, the cross-QTFRs $T_{x_{k}, x_{l}}(t, f)$ corresponding to an orthonormal basis $\left\{x_{k}(t)\right\}$ of the linear space of finite-energy analytic signals are an orthonormal basis of $L_{2}\left(\mathbb{R} \times \mathbb{R}_{+}\right)$. Hence, any square-integrable function $F(t, f)$ defined for $f>0$ can be expanded as in (10), where the expansion coefficients are given by $c_{k l}=\left\langle F, T_{x_{k}, x_{l}}\right\}$. In the case of an affine QTFR completeness of the cross-QTFRs requires the additional condition (11).

Least-Squares Signal Synthesis. An important implication of unitarity is the applicability of a standard method for least-squares signal synthesis. The signal synthesis problem $[13,14]$ is the calculation of a signal $x_{\mathrm{opt}}(t)$ whose QTFR is closest to a given square-integrable TF function ("model") $M(t, f)$,

$$
x_{\text {opt }}(t) \triangleq \arg \min _{x}\left\|M-T_{x}\right\| .
$$

Assuming unitarity of the QTFR $T$, the signal synthesis result $x_{\text {opt }}(t)$ can be derived as follows [13]:

(i) The matrix $\mathbf{H}$ with elemente $H_{k l}=\left\langle M, T_{x_{k}, x_{l}}\right\rangle$ is calculated, where $x_{k}(t)$ with $k=1, . ., K$ is an orthonormal basis of the underlying linear signal space ${ }^{4}$.

(ii) The Hermitian part of $\mathbf{H}$ is formed, $\mathbf{H}_{h}=\frac{1}{2}\left[\mathbf{H}+\mathbf{H}^{*}\right]$, where $\mathbf{H}^{*}$ denotes the complex conjugate transpose of $\mathbf{H}$.

${ }^{3}$ The inner product is defined as $\left(x_{1}, x_{2}\right\rangle=\int_{t} x_{1}(t) x_{2}^{*}(t) d t$ for signals and $\left(T_{1}, T_{2}\right)=\int_{t} \int_{f} T_{1}(t, f) T_{2}^{*}(t, f) d t d f$ for QTFRs.

In practice, this is a space of finite-length, discrete-time signals, so that the dimension $K$ is finite. 
(iii) The largest eigenvalue $\lambda_{1}$ and the corresponding eigenvector $e_{1}$ of $\mathbf{H}_{h}$ are calculated.

(iv) If $\lambda_{1}>0$, the signal synthesis result is given by

$x_{\text {opt }}(t)=\sqrt{\lambda_{1}} e^{j \phi} e_{1}(t)$ with $e_{1}(t)=\sum_{k=1}^{K}\left(e_{1}\right)_{k} x_{k}(t)$,

where $\phi$ is an arbitrary constant phase and $\left(e_{1}\right)_{k}$ denotes the $k$ th element of the eigenvector $e_{1}$. If $\lambda_{1} \leq 0$, the signal synthesis result is $x_{\text {opt }}(t)=0$.

\section{THE AFFINE-HYPERBOLIC CLASS}

The affine-hyperbolic class (AHC) is defined as the intersection of the AC and the HC. QTFRs of the AHC belong to both the AC and the HC, and thus satisfy the scale invariance, time-shift invariance, and hyperbolic time-shift invariance properties [5]. An affine-hyperbolic QTFR can be expressed by either of the normal forms (1) and (2). It can be shown that these normal forms reduce to

$$
\begin{aligned}
T_{x}(t, f) & =\int_{\nu} B\left(\frac{\nu}{f}\right) G_{T}\left(\frac{\nu}{f}\right) U_{x}\left(f A\left(\frac{\nu}{f}\right), \nu\right) e^{j 2 \pi t \nu} d \nu \\
& =\int_{\beta} G_{T}(\beta) V_{x}\left(\ln \left(\frac{f}{f_{r}} B(\beta)\right), \beta\right) e^{j 2 \pi t f \beta} d \beta
\end{aligned}
$$

where $A(\beta)=\frac{\beta}{2} \operatorname{coth}\left(\frac{\beta}{2}\right), B(\beta)=\frac{\beta}{2} / \sinh \left(\frac{\beta}{2}\right)$, and $G_{T}(\beta)$ is a one-dimensional "kernel" characterizing the QTFR $T$. This corresponds to the following special forms of the AC and HC kernels in (1) and (2), respectively:

$$
\begin{gathered}
\Gamma_{T}(b, \beta)=B(\beta) G_{T}(\beta) \delta(b-A(\beta)) \\
\Phi_{T}(b, \beta)=G_{T}(\beta) \delta(b+\ln B(\beta)) .
\end{gathered}
$$

The Bertrand distribution $P_{x}(t, f)$, obtained with $G_{T}(\beta)=$ 1 , is the "central" member of the AHC. Any other AHC member $T_{x}(t, f)$ can be derived from the Bertrand distribution via a frequency scaled convolution with respect to time,

$$
T_{s}(t, f)=f \int_{t^{\prime}} g_{T}\left(f\left(t-t^{\prime}\right)\right) P_{x}\left(t^{\prime}, f\right) d t^{\prime}, \quad f>0,
$$

where $g_{T}(a)$ is the inverse Fourier transform of $G_{T}(\beta)$ in (12).

Regularity and Unitarity. An affine-hyperbolic QTFR is reqular if its AHC kernel satisfies $G_{T}(\beta) \neq 0$. The inverse kernel in the AC framework is then given as

$$
\Gamma_{T}^{-1}(b, \beta)=\frac{1}{B(r(\beta)) G_{T}(r(\beta))} \delta\left(b-\frac{\beta}{r(\beta)}\right)
$$

where $r(\beta)=2 \tanh ^{-1}\left(\frac{\beta}{2}\right)$. The inverse HC kernel is

$$
\Phi_{T}^{-1}(b, \beta)=\frac{1}{G_{T}(\beta)} \delta(b-\ln B(\beta))
$$

An affine-hyperbolic QTFR is unitary if and only if its AHC kernel is unimodular, $\left|G_{T}(\beta)\right|=1$. The Bertrand distribution $P_{x}(t, f)$ is unitary since $G_{P}(\beta)=1$.

\section{CONCLUSION}

We have discussed the QTFR properties of regularity and unitarity in the framework of the affine and hyperbolic QTFR classes. The regularity property expresses the QTFR's "reversibility." Important implications of regularity are the following: (i) From a regular QTFR, the signal can be recovered up to a constant phase factor. (ii)
Any quadratic signal representation can be derived from a regular QTFR. (iii) The optimum detection statistic for detecting a Gaussian random signal can be formulated ae a TF correlation involving the QTFR of the observed signal. (iv) A linear signal traneform induces a linear QTFR transform. (v) The crose-QTFRs of linearly independent signals are linearly independent. In the case of a hyperbolic QTFR, a signal basis induces a QTFR basis.

The property of unitarity is equivalent to the validity of Moyal's formula. Unitarity always implies regularity. Two further implications of unitarity are as follows: (i) The cross-QTFRs of orthonormal signals are orthonormal. In the case of a hyperbolic QTFR, an orthonormal signal basis induces an orthonormal QTFR basis. (ii) The problem of least-squares signal synthesis can be solved by a standard method in which the optimum signal is given by the dominant eigenvector of a Hermitian matrix.

Examples of unitary affine or hyperbolic QTFRs are the (generalized) Wigner distribution, the Bertrand distribution, and the (generalized) Alteg-Marinovic distribution. QTFRs involving a smoothing operation (like the scalogram or the hyperbologram) are non-regular.

\section{References}

[1] J. Bertrand and P. Bertrand "Affine time-frequency distributions," in Time-Frequency Signal Andygis-Methods and Application, ed. B. Bo

[2] O. Rioul and P. Flandrin, "Time-scale energy distributions: A general class extending wavelet transforms," IEEE Thane. Sig. Proc., vol. 40, no. 7, pp. 1746-1757, July 1992.

[3] P. Flandrin, "Sur une classe générale d'extensions affines de la distribution de Wigner-Ville," 1Stme Coll. GRETSI, Juan-les-Pins, France, Sept. 1991.

[4] A. Papandreou, F. Hlawatsch, and G.F. BoudreauxBartels, "A unifled framework for the Bertrand distribution and the Altes distribution: The new hyperbolic class of quadratic time-frequency distributions," Proc. IEEE Symp. Time-Frequency and Time-Scale Analysis, Victoria, Canada, pp. 27-30, Oct. 1992.

[5] A. Papandreou, F. Hlawatsch, and G.F. BoudreauxBartele, "Quadratic time-frequency distributions: the new hyperbolic class and its intersection with the affine class," Sixth SP Workhop on Statiotical Signal and Array Proce, Victoria, Canada, pp. 26-29, Oct. 1992.

[6] A. Papandreou, F. Hlawatech, and G.F. BoudreauxBartels, "The hyperbolic class of quadratic time-frequency representations, "submitted to IEEE Thans. Sig. Proc.

[7] R. A. Altes and E. L. Titlebaum, "Bat signals as optimally Doppler tolerant waveforms," J. Acout. Soc. Am, vol. 48, no. 4 , pp. 1014-1020, Oct. 1970.

[8] P. Flandrin "Scale-invariant Wigner spectra and selfaimilarity, ${ }^{,}$Proc. EUSIPCO-90, Barcelona, Spain, pp. 149 152, Sept. 1990.

[9] F. Hlawatsch, "Regularity and unitarity of bilinear timefrequency signal representations, IEEE Than. Inform. The ory, vol. 38, no. 1, pp. 82-94, Jan. 1992.

[10] H.L. Van Trees, Detection, Estimation, and Modulation Theory, Part III. New York: John Wiley \& Sons, 1971

[11] P. Flandrin, "A time-frequency formulation of optimum detection," IEEE Thans. Acout., Speech, Signal Proc., vol. ASSP36, pp. 1377-1384, Sept. 1988.

[12] F. Hlawatsch, "Duality and classification of bilinear timefrequency signal representations," IEEEE Trans. Sig. Proc. vol. 39, no. 7, pp. 1564-1574, July 1991 .

[13] F. Hlawatuch and W. Krattenthaler, "Bilinear signal aynthesis," IEEE Trane. Sig. Proce, vol. 40, no. 2, pp. 352-363 Feb. 1992.

[14] G.F. Boudreaux-Bartels and T.W. Parks, "Time-varying fltering and signal estimation using Wigner distribution synthesis techniques," IEEE Thans. Acout., Speech, Signal Proc., vol. ASSP-34, pp. 442-451, June 1986. 